\title{
A Framework Algorithm to Compute Optimal Asset Allocation for

\author{
Retirement with Behavioral Utilities
}

\author{
Aparna Gupta and Walter Murray* \\ Management Science and Engineering, Stanford University, Stanford, CA 94305
}

\begin{abstract}
The question of optimal strategic asset allocation for investors with behavioural utilities saving for retirement is addressed. To date this problem has been studied assuming that an investor is rational in the sense when making investment decisions the preference relation of the investor satisfies all the axioms of choice. Research in behavioural science indicates that investment related decisions of many people do not satisfy the axioms of choice. Our interest is in developing a platform that allows the use of a broader class of utilities that may or may not satisfy the axioms of choice. Such utilities may not be convex. Our interest is in developing a framework algorithm that enables a user considerable flexibility in how their needs may be specified. For illustrative purposes a binomial tree is used to model asset returns, although the method developed can be used with more elaborate models.
\end{abstract}

${ }^{*}$ Work supported by the National Science Foundation grant CCR-9988205 and Office of Naval Research grant N00014-96-1-0274 


\section{Introduction}

The investment problem of how an investor should invest for meeting long-term financial targets and goals is viewed as an important problem and has been the subject of considerable research $[34,27,29,9,43,31]$. In such studies there are assumptions made about the planner's preferences, assets available for investment and rules for their price evolution. Here we develop a platform where behavioural aspects of the problem can also be addressed by obtaining the optimal investment decisions for investors with a class of behavioural utilities and/or adding behavioural constraints to the problem. The traditional utilities can also be solved with this approach. The main emphasis of this financial planning problem is on retirement planning.

For expository purposes the asset-price evolution is modeled using a binomial-tree model (Sharpe [35]). Our framework is flexible in the choice of a model, for example, we can accommodate an irregular pattern of branching in the tree, variation in the number of outcomes in each time period, and irregular events and constraints in the pattern of investment. It will be seen later that the use of trees, while not essential, does have attractive features for the particular approach we take. The investment strategy is posed as the solution of a nonlinearly constrained optimization problem, which is solved using a general-purpose algorithm. One of the issues of interest is whether a general-purpose algorithm, which allows more model flexibility, could successfully solve large problems that arise with long-term financial planning problems.

The organization of the paper is as follows. We will begin with describing the modeling of preferences of a decision-maker, with a focus towards behavioural issues. This is followed 
by a mathematical formulation of the long-term financial planning problem. Solution methods suitable for solving large-scale nonlinearly constrained optimization problems arising in the financial planning problem are discussed next. We will then present solutions for a representative set of utility functions, including some that do not satisfy the economic rationality assumptions. The article is concluded with some summarizing comments and future directions for research.

\section{$2 \quad$ Modeling Preferences}

\section{$2.1 \quad$ Utility Theory}

An individual faced with a decision chooses from a set of alternatives, say $X$. Among all elements of $X$ the decision-maker weighs the merits and de-merits of each option and attempts to select the option that suits her "best." This requires a precise definition of "best."

In more rigorous terms, we need to define an ordering for the set of alternatives that makes it possible to compare every element of the set with every other element. We denote this ordering by ' $\succeq$.' If $x, y \in X, x \succeq y$ will imply that the decision-maker prefers $x$ to $y$ and $x \succ y$ would imply that $x$ is strictly preferred to $y$.

The preference relation defined above is said to be rational if it satisfies the axioms of choice. These axioms are reflexivity, completeness and transitivity and are defined as follows (see Varian [42], MasColell [26]) :

Reflexivity For all $x \in X$ we have $x \succeq x$. 
Completeness For all $x, y \in X$ we have either $x \succeq y, y \succeq x$ or both.

Transitivity For all $x, y, z \in X$, if $x \succeq y, y \succeq z$ then $x \succeq z$.

The imposition of these axioms has enormous implications in practical terms. Although at first sight these axioms may look reasonable, it is worth taking a closer look. Reflexivity is the weakest and most acceptable axiom. The completeness axiom implies that the decisionmaker has done the introspection of all the alternatives, however far removed they may be from ones realm of common experience. It is easy to imagine that this may need serious work and reflection on one's preferences and may in some cases be almost impossible. The transitivity axiom on the other hand implies that in a sequence of pairwise choices there is no possibility of cycles. This implies that irrespective of the manner in which the options are framed or presented, the decision-maker is mature enough to be able to rank all of them in an order that contains no cycles. Besides the above three axioms of choice, preference relations are assumed to satisfy more axioms such as monotonicity, continuity and convexity of preferences.

\subsection{Normative vs. Descriptive(Behavioral) Choice}

The axioms of choice listed in the previous section form the normative theory of choice. A decision-maker who satisfies these axioms is said to be rational and it is possible to represent the preferences in terms of a utility function, which is a map from the set of alternatives to the real line. Further assumptions of continuity, monotonicity and convexity of the preference relation imply that the utility function obtained is a continuous, increasing and concave function. Most research in economics and finance have developed elegant and elaborate 
theory on the basis of the assumption that the decision-makers' preferences satisfy these axioms.

As was observed in the previous section, it may be a difficult proposition for an average decision-maker to satisfy all the axioms of choice. Following this thought many behavioural scientists and economists have attempted to understand the decision-making processes among ordinary people through experiments and studies of various kind (see Wright [44], Bell [1], Simon [37], Rubinstein [33]). These attempts also try to quantify the choice making process, but try to be parsimonious in their assumptions. They constitute what is called the descriptive theory of choice. The five major phenomena of choice that violate the standard model of normative theory are listed as : framing effects, nonlinear preferences, source dependence, risk seeking and loss aversion. These have been confirmed in a number of experiments, with both real and hypothetical payoff and are defined as follows in Tversky \& Kahnemann [41, 40], Tversky [39].

Framing effects Lack of description invariance implying that variations in the framing of options yield systematically different preferences.

Nonlinear preferences The expectation principle of utility theory states that utility of risky prospects is linear in outcome probabilities. However, experimental evidence indicates that people tend to transform probabilities nonlinearly, overweighing small probabilities and underweighing moderate and high ones.

Source dependence Willingness to bet on an uncertain event depends not only on the degree of uncertainty but also on its source. 
Risk seeking As opposed to the generally assumed risk aversion in economic analysis, in certain situations people prefer more risk to less. For instance, people prefer a small probability of winning a large prize over the expected value of that prospect.

Loss aversion Carriers of value are gains and losses defined relative to a reference point and the losses loom larger than the gains. Implying that an amount of loss elicits more 'unhappiness' than the same amount of gain elicits 'happiness.'

In behavioral finance researchers have made an attempt to understand and explain the investment style and preferences of individual investors and other market characteristics from a descriptive standpoint $[2,20,22,23,38,41]$. It is hoped that these descriptive studies will help bridging the gap between the theories developed on the rationality hypothesis and what is observed in practice. Bridging this gap will help experts in providing better advice and 'educate' people to make better decisions.

\section{The Portfolio Problem}

The portfolio problem typically contains elements like how much to invest, what to invest in, what point in ones life-cycle should one invest in what assets, how should one invest in order to have maximum tax benefits over the life-cycle, etc. An investor saves from current earnings for future consumption and invests the savings in investment vehicles, broadly classified into asset classes like stocks, bonds, gold, real estate, etc. The investor evaluates the performance of the investments using some criterion, we call this the investor's objective, say $U($.$) , which$ may be a function of returns or wealth or any other aspect of the investment the investor values. The portfolio problem may be described in broad terms as determining the set of 
decisions to maximize this objective function $U($.$) .$

Most work done so far has been within a normative framework, assuming that the planner is economically rational. This implies that the utility function representation of preferences is possible and the utility function is a smooth, increasing, concave function. The problem can then be framed as a finite-horizon continuous/discrete-time stochastic control problem, solvable by optimal stochastic control techniques in the continuous-time case or dynamic programming techniques in the discrete-time case. This is the classical investment-consumption problem, which was first solved as a discrete-time problem by Samuelson [34]. Merton [27, 28] posed the problem as an optimal stochastic control problem in continuous-time. Following this work, several researchers have solved the investment-consumption problem with additional features and relaxed assumptions.

Among the more recent works, Brennan et al [8] modeled the rate of return on the investment portfolio to be governed by a set of state variables. The nonlinear partial differential equation from the Hamilton-Jacobi-Bellman (HJB) equation for the value function was solved using implicit finite difference approximation. Karatzas et al [19] solved the optimal portfolio choice problem in continuous-time as an optimal control problem and obtained a closed form solution of the HJB equation for general strictly increasing and concave utilities. Duffie and Zariphopoulou [12] generalized the above by introducing a stochastic income process in the problem that is undiversifiable. They obtained a weak solution to the HJB equation, referred to as a viscosity solution. Bodie et al [7] considered a life-cycle model with an optimal investment choice, choice of labor and consumption over the lifetime of the planner. They pose the problem as an optimal control problem in continuous-time and solve 
it by assuming the wages are perfectly hedged. Relations are drawn between the optimal investment choice and the human capital of the planner over the planning period. Shoven [36] considered an allocation-location problem of wealth in pension and savings accounts. Solutions for the continuous-time formulations for the problem are hard to obtain in complex settings, such as, in case of relaxed assumptions on properties of the objective (utility) function and constraints. Moreover, discrete-time dynamic programming techniques suffer from the well-known curse of dimensionality, due to the rapid increase in number of states with the increase in number of sources of uncertainty and time-periods.

In recent years stochastic programming has developed as a powerful technique to solve multi-period stochastic optimization problems [5], and specifically its application in financial decision-making problems (see Kouwenberg and Zenios [21] and Mulvey et al [30] for an excellent overview). Birge [4], Dantzig and Infanger [11], Infanger [17] approach the problem in the stochastic optimization framework. The problem is posed as a large linear program and solved using decomposition techniques, such as Bender's decomposition. Nonlinear objective and constraints may also be considered in the stochastic programs, where deterministic Lagrangian-based methods may be employed [5, 3]. Stochastic programming allows the flexibility of including several sources of uncertainty in the decision problems. However, in a multi-stage stochastic program, the computational effort increases prohibitively quickly with increase in the number of decision stages.

Mathematically, a discrete-time version of the long-term financial planning problem may be written as

$$
\max _{\mathbf{x}_{\mathbf{t}}, C_{t}, t=0, T-1} U \equiv \sum_{t=0}^{T} E\left[U_{t}\left(W_{t}, C_{t}, \mathbf{R}_{\mathbf{t}}, \ldots\right)\right]
$$


where $T$ is the planning horizon, $W_{t}$ is the wealth at time t, $C_{t}$ is the consumption at time $\mathrm{t}, \mathbf{R}_{\mathbf{t}}$ is a vector of returns on the asset classes. $U_{t}($.$) is the utility function at time t$ for wealth, return on assets, consumption or any other properties at each decision point $t . \mathbf{X}_{\mathbf{t}}$ is the vector of wealth invested in each asset class at time $t$, i.e., if there are $N$ asset classes, $\mathrm{X}_{\mathbf{t}}$ will be a vector of size $N$.

The above is a general form for the problem. Since our focus here is on asset allocation for long-term goals, such as, for retirement, we disregard consumptions and optimize investment decisions for wealth accumulated at retirement. The problem is simplified by taking investor's utility to be a function of only the wealth at the planning horizon, $T$. The typical planning horizon ranges from 10-25 years, where at least yearly asset-allocation decisions are made. This makes dynamic programming approach unfavorable. The computational effort for a multi-stage stochastic programming formulation may also become prohibitive for these many decision stages. The solution approach should also be capable of incorporating behavioural utilities and constraints. We describe the modeling in detail next.

\subsection{Modeling the Portfolio Problem}

An investment portfolio's growth is driven by how much we save and invest, what we invest in, how long we invest, what return we earn and how much of that return we surrender to investment costs (transaction costs) and to taxes. As an individual investor we usually have a great amount of control over every factor above, except one, the asset-returns.

The returns we get on our investments are uncertain. The past performances may be indicative of future performance, but in no way are fully predictive. This fact makes modeling 
the asset-return dynamics particularly challenging. In the following sections we describe a model we use for asset-return dynamics and formulate the optimal investment/consumption problem in terms of it. Although the platform developed here allows far more general models, for expository purposes we describe a simple binomial-tree model.

Models for asset-return or price dynamics can be classified in two groups, continuous-time models and discrete-time models. In continuous time, asset-price dynamics are modeled as being governed by a stochastic differential equation, which in turn is driven by an underlying brownian motion or a set of factors that are driven by brownian motion. Various additional features, like mean-reversion and stochastic volatility are introduced to add realism. These models have had varying degrees of success in their applications, however, they become quite complicated and analytical solutions may not be possible. (See Duffie [12], Lamberton [24] for more details on continuous-time models.)

In discrete-time models the asset-return dynamics are primarily governed by a transition rule. A discrete-time model could in some cases be considered to be a time-discretization of a continuous-time stochastic differential equation model obtained by discretizing the brownian motion or other sources of randomness therein. There may not always be a one-to-one map for all discrete-time models to a continuous-time model since this depends on whether or not there is a limit of the discrete-time transition rule as the time-grid size shrinks to zero.

The discrete-time models are often modeled as discrete-time, discrete-space asset-return models with a finite number of states. These models are often represented by a 'tree.' If each state at each time is depicted by a node, an arc can be drawn from this node to every node at the next time period to which transition is possible. 


\subsubsection{Asset-price Dynamics - Binomial Tree Set-up}

We have chosen a discrete-time, discrete-space asset-return dynamics modeling approach for our problem. At each point of time in this model, transition is possible to two states for asset-returns, we call one of the states 'up' and the other 'down.' The 'up' refers to a return greater than 1, or in other words, the price of the asset goes up, and the 'down' refers to the return being less than 1 . At first sight this may look like a simplistic approach, but this model has enjoyed enormous success due to its ease of use and yet its capacity to capture important characteristics of the problem $[35,10,33]$. We consider it to be equally probable for the returns to go up as to go down. Due to the two-way branching at each node, this model is commonly known as the binomial-tree model. It is a version of a simple symmetric random walk, the limit of which is the brownian motion, as the size of time-interval shrinks to zero. This further entails that the asset-price process dictated by the binomial-tree model is a geometric brownian motion in the limit (see Luenberger [25] and Ross [32] for details).

We consider two asset-classes, a risky one and a riskfree one. At each point of time the risky asset's return goes up (to $S_{u}$ ) with a probability $p=1 / 2$ and down (to $S_{d}$ ) with probability $1-p=1 / 2$, whereas the return of the riskfree asset stays a constant, say at $R_{f}$. Therefore, if one invests $W_{s}$ dollars in the risky asset and $W_{b}$ in the riskfree asset in a period, the wealth one would have in the next period will be $W_{s} * S_{u}+W_{b} * R_{f}$ when an 'up' occurs and $W_{s} * S_{d}+W_{b} * R_{f}$ when a 'down' occurs. See figure 1 for pictorial description of the binomial-tree model. 


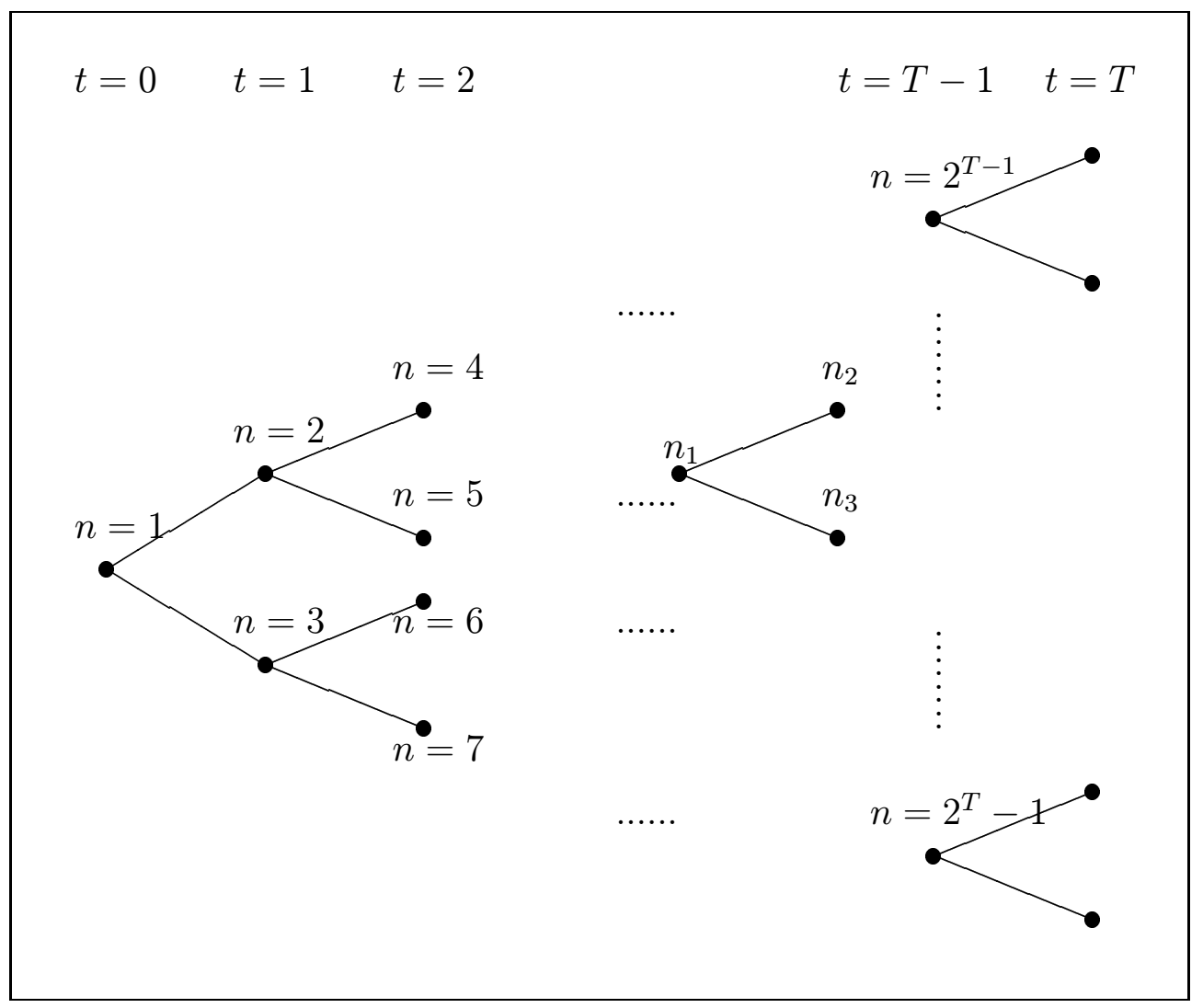

Figure 1: Ordering of nodes in the binomial tree.

\subsubsection{Optimal Asset Allocation Problem}

With the binomial-tree model the optimal asset allocation problem can be formulated as follows. Given a time-horizon, $T$ for the planning problem at each time point, $t$ lying in $\{0,1, \ldots, T-1\}$, a decision regarding the amount of wealth to invest in the two asset-classes needs to be made. Given the investor knows the state of the world at $t=0$, there are $2^{T}-1$ nodes between $t=0$ and $t=T-1$, each representing a decision variable, namely how much of wealth to invest in the risky asset and the riskfree asset. The nodes are ordered as indicated in figure 1. Let each decision variable be denoted by $X_{t, n}$, where $t$ is the time period and $n$ is the number of the node in the ordering. There is also an amount of wealth associated with each node in the tree, denoted by $W_{t, n}$ by the same convention as for the 
investment decision variable. This is the wealth the investor has at that node given that she starts with a wealth $W_{0}$ at $t=0$ and invests according to a particular set of values for the decision variables, $X_{t, n}$. The relationship between the decision variables $X_{t, n}$ and state variables $W_{t, n}, S_{u}, S_{d}$ and $R_{f}$ can be written as follows:

$$
\begin{gathered}
W_{t+1, n_{2}}=X_{t, n_{1}} * W_{t, n_{1}} * S_{u}+\left(1-X_{t, n_{1}}\right) * W_{t, n_{1}} * R_{f} \\
W_{t+1, n_{3}}=X_{t, n_{1}} * W_{t, n_{1}} * S_{d}+\left(1-X_{t, n_{1}}\right) * W_{t, n_{1}} * R_{f}, t=0 . . T-1, n_{1}=1 . .2^{T}-1,
\end{gathered}
$$

where (1) is for an 'up' and (2) for a 'down.' $\left\{X_{t, n}, t=0 . . T, n=1 . .2^{T}-1\right\}=\mathrm{X}$ are taken to be the fraction of ones wealth at time $t$ and node $n$ one invests in the risky asset. This makes $\left(1-X_{t, n}\right)$ the fraction of ones wealth invested in the riskfree asset at time $t$ and node $n$. The relation between the nodes $n_{1}, n_{2}$ and $n_{3}$ is indicated in figure 1 . The choice of decision variables to be fraction of wealth simplifies their bounds to be a constant for all nodes.

Utility is from the wealth at the planning horizon, since the investor does not consume prior to the planning horizon. The optimal asset allocation problem is written as follows:

$$
\begin{array}{r}
\max _{X} \sum_{m=2^{T-1}}^{2^{T}-1}\left[p * U\left(X_{T-1, m} * W_{T-1, m} * S_{u}+\left(1-X_{T-1, m}\right) * W_{T-1, m} * R_{f}\right)+\right. \\
\left.(1-p) * U\left(X_{T-1, m} * W_{T-1, m} * S_{d}+\left(1-X_{T-1, m}\right) * W_{T-1, m} * R_{f}\right)\right],
\end{array}
$$

where $p$ is the probability of each node at the planning horizon. The summation is over nodes one period prior to planning horizon (see figure 1).

The problem so formulated is a nonlinear programming problem with bounds on variables. This can be seen by substituting the $W_{T-1, m}$ term in the expression at which $U($.$) is evaluated$ by (1) or (2). Nonlinearity is further enhanced due to nonlinearity in the function $U($.$) . The$ smoothness properties of our objective are largely dependent on the smoothness properties 
of the utility function $U($.$) , since the expression at which the utility is evaluated is a smooth$ function of the decision variables. However, even if $U$ is a differentiable function, it is very cumbersome to explicitly compute the gradients. Software packages like ADIFOR may be used to generate the derivative code [6], however these will not be efficient since in executing the chain rule they will need to traverse the entire tree for differentiation with respect to each variable. An alternative would be to use optimization methods that either use only function value for determining descent direction or perform a finite-difference approximation for the gradient of the objective to compute a descent direction [16]. Alternately, we could reformulate the problem so that exact derivatives become easier to compute. This is explored in the next section.

In our problem formulation we permit no short sale, i.e. borrowing of assets is not allowed. This restriction translates to bounds on the decision variables. These bounds reflect regulatory constraints on positions the planner can take on the asset classes. It would not seriously alter either the nature of the problem or the method of solution if short selling was allowed.

\subsection{Modeling Variations}

In this section we first consider some variations in the modeling of the optimization problem and then consider some variations in the model for the investment problem. 


\subsubsection{Wealth as Variable}

Earlier we formulated the optimal investments problem as a nonlinear programming problem in terms of the decision variables, $X$. This formulation makes the computation of the objective gradients expensive and cumbersome. It is possible to rectify this by considering the wealth at each node to be a variable in the optimization problem. Since the wealth values at each node are fully determined once the investment decisions $X$ are known, we need to include constraints that impose this characteristic of the investment problem. With this change there will be $2 *\left(2^{T}-1\right)$ variables in the new optimization problem. In the terminology of optimal control, the wealth variables are the state-variables of the problem, whereas $X$ 's are the control variables. In a typical stochastic control problem the number of state variables is much larger than the number of control variables, but in our case they are the same in number, hence treating them both as variables is feasible. However, we now need to solve a nonlinearly constrained optimization problem. The nonlinear constraints of the problem are given by (1) \& (2), where $X, W_{t, n_{1}}, W_{t+1, n_{2}}$ and $W_{t+1, n_{3}}$ are now variables for all $t, n_{1}, n_{2}, n_{3}$ relevant indices. This implies that the problem now has as many nonlinear constraints as there are decision variables, i.e. $2^{T}-1$ of them. These constraints are very structured, they are bilinear, i.e. each term in the left-hand-side of the constraint is a product of two variables, and each constraint involves only three variables. These observations imply that the constraint Jacobian of the constraint function is very easy to compute, and is sparse and structured. All these properties can be exploited in an optimization algorithm. The most attractive advantage of this modeling variation is that now it is possible to compute all the objective gradients with great ease. It is in fact, also possible to compute the 
exact Hessian of the objective and constraint function, which enables the Hessian of the Lagrangian function to be computed. Like the Jacobian matrix, the Hessian of the Lagrangian is sparse and highly structured. In the original formulation although the Hessian of the objective is half the size, it is dense and hard to compute. The gains in efficiency from this modeling variation greatly outweigh the losses from an increase in problem size.

A question that arises at this point is should the wealth at nodes at the planning horizon, $T$ also be treated as variables? If we do so, the number of nonlinear constraints increases to $2^{T}-1+2^{T}$, which is an increase of $2^{T}$, and the number of variables increases to $2 *\left(2^{T}-1\right)+2^{T}$, which is also an increase of $2^{T}$. By doing so the objective function of the problem becomes:

$$
\max _{X, W} \sum_{m=2^{T}}^{2^{T+1}-1}\left[p * U\left(W_{T, m}\right)\right]
$$

where $\mathrm{p}$ is the probability of each final node, and $W=\left\{W_{t, n}, t=0 . . T, n=1 . .2^{T+1}-1\right\}$. Clearly, this further reduces the nonlinearity of the objective. However, we observe that the benefits from this change do not outweigh the loss of efficiency from an increased number of variables and constraints. This modeling approach may be desirable if we need to impose some additional bounds on the wealth at the planning horizon.

\subsubsection{Non-Uniform Time Periods}

The investment problem is solved on a rolling basis, that is, each time an investment decision needs to be made the optimization problem is solved taking the currently owned wealth as the starting wealth of the problem. The optimal decision for $t=0$ is implemented. This implies that the optimal decisions suggested for subsequent periods are not of direct relevance to the planner. Such usage of the model suggests that there should be a finer time-resolution in 
the beginning of the planning horizon compared to later. In other words, the model for the problem should have shorter time-periods in the beginning of the problem, which become progressively larger towards the end.

This change in the model of the investment problem does not structurally change the character of the optimization problem. The parameters of the problem change, namely the asset returns for the periods vary and depend on the length of each period.

\section{Method for Solution}

In seeking a method to solve our problem we did not want the method to be too specific to a particular model for asset-return dynamics. What we wanted to establish is that a powerful general purpose algorithm could solve our current models. By using a general purpose algorithm we will not need to switch algorithms for additional complexity introduced to the model.

The problem as formulated is a nonlinearly constrained optimization problem. The expression for wealth at the planning horizon, at which the utility is evaluated, is a nonlinear function of wealth and investment decisions at the prior period. Nonlinearity of the utility function further enhances the nonlinearity of the objective. Smoothness properties of our objective are largely dependent on the smoothness properties of the utility function $U($.$) ,$ since the expression at which the utility is evaluated is a smooth function of the decision variables.

Sequential Quadratic Programming (SQP) methods are considered the most robust and efficient algorithms for solving nonlinearly constrained optimization problems. They are 
iterative procedures and can be viewed as a generalization of Newton's method to the (inequality) constrained problem. The basic idea is to construct a constrained quadratic model around the current iterate, $x_{k}$. Maximization of the quadratic model gives an update $p_{k}$ that approximates the error $\left(x^{*}-x_{k}\right)$, where $x^{*}$ is a local optima of the objective function, $F$. The new iterate determined as $x_{k+1}=x_{k}+\alpha_{k} p_{k}$, where $\alpha_{k}$ is usually determined by a line search method applied to a merit function.

In other words, SQP methods solve a sequence of quadratic programs (QPs). The QP constraints are linearization of the nonlinear constraints at $x_{k}$. The objective is a quadratic approximation to the Lagrangian. The $k$ th QP subproblem can be stated as

$$
\begin{gathered}
\text { QP : } \max _{p} g_{k}^{T} * p+p^{T} * H_{k} * p \\
\ni \quad J_{k} * p=-c_{k},
\end{gathered}
$$

where $g_{k}=\nabla F\left(x_{k}\right), F\left(x_{k}\right)$ is the objective, $J_{k}$ is the Jacobian of the constraint vector $c\left(x_{k}\right)$, and $H_{k}$ is an approximation to the Hessian of the Lagrangian. Most SQP methods do not require exact second derivatives because these may be unavailable or expensive to compute. Exact Hessians also give rise to deep technical issues due to nonconvexity. Typically the Hessian of the Lagrangian is approximated using a quasi-Newton update and the approximation is forced to be positive definite. Consequently, QP has a unique solution, which has important theoretical and numerical implications. SQP methods incorporating exact second derivatives are under development, but at this time reliable software is not available.

Any algorithm for constrained optimization needs to ensure that the algorithm converges to a point that is both feasible and a constrained minimizer. Therefore, to measure how good the next iterate is, simply looking at the objective is not enough because that does 
not say anything about how close the iterate is to the feasible set. The standard approach today is to define a merit function, which combines both the objective and the constraint violations into one function. For this reason, merit functions are closely related to penalty functions. The $l_{1}$ merit function $M(x)=F(x)+\rho *\|c(x)\|_{1}$, is a popular merit function. SNOPT uses a complicated version of the augmented Lagrangian. After $p$, the solution of the QP, has been determined, we search along it to find a step length $\alpha$. Most convergence proofs for SQP methods rely on proving a sufficient increase in the merit function at each iteration.

We used the SNOPT [14] software package, a Fortran based package for nonlinear programming from the Systems Optimization Laboratory at Stanford University. SNOPT implements an SQP algorithm [15]. It maximizes a nonlinear function subject to bounds on the variables and sparse nonlinear constraints. SNOPT finds local solutions, and ideally any nonlinear functions should be smooth and users should provide gradients, but this is not essential. Any unknown gradients are estimated by finite differences. Discontinuities in the function gradients can often be tolerated if they are not too close to an optimum.

A feature of SQP methods is the need to approximate the Hessian of the Lagrangian. SNOPT obtains a compact representation of this matrix using a limited-memory quasiNewton approximation. No assumption is made that this Hessian is sparse. It is assumed that the Jacobian is sparse and this is stored in compact form (only the nonzero elements). The linear systems arising in computing search directions are solved using sparse matrix factorizations. Each QP subproblem is iteratively solved using a quadratic programming package with several features that improve the efficiency of an SQP algorithm. SNOPT 
is most efficient if only some of the variables enter nonlinearly, or if the number of active constraints (including simple bounds) is nearly as large as the number of variables. It requires relatively few evaluations of the problem functions. Hence, it is especially effective if the objective or constraint functions (and their gradients) are expensive to evaluate. In cases where objective and constraint functions are structured, the structure is automatically exploited.

These features of SNOPT make it suitable for our problem. Not only does the constraint Jacobian of our problem have the structure that SNOPT can exploit, the number of bounds and constraints active at the solution is comparable to the number of variables. The current version of SNOPT does not use second derivative information of the objective or constraints. As pointed out earlier, it is possible to compute the exact Hessian of the objective and constraint function for our problem, which enables the Hessian of the Lagrangian function to be computed. Further, like the Jacobian matrix, the Hessian of the Lagrangian is sparse and highly structured. Being able to use these additional features of our problem in the solution procedure will give us a much more efficient solution method.

It is worth noting that all methods for obtaining a solution for nonlinear optimization problems are iterative and hence will almost always benefit from starting from a good initial point. In the model and solution method developed here it is not only possible to supply a good initial point, but also one that is feasible. It also improves the efficiency of SNOPT if the number of active constraints at the initial point is large. Here we can compute a feasible vertex. 


\subsection{An efficient Null-space Representation}

A key aspect of an SQP method is the representation of a basis for the null space of the constraint Jacobian. Within SNOPT the Jacobian is divided as follows

$$
J=\left[\begin{array}{ll}
B & S
\end{array}\right]
$$

where $B$ is a nonsingular matrix. The null space may then be represented by

$$
Z=\left[\begin{array}{ll}
-B^{-1} S & I
\end{array}\right]
$$

The matrix $Z$ is not computed explicitly. Rather a sparse LU factorization of B is determined and then where $Z^{T} v$ or $Z * v$ is required such a product may be formed knowing $\mathrm{S}$ and the LU factors. In the problems of interest to us the structure of $\mathrm{J}$ is extremely special. Assuming the $W$ (wealth) variables are listed first, then $J$ has the form

$$
[D \mid T]
$$

where $D$ is a diagonal matrix and $T$ is bidiagonal. Consequently, no factorization is required and an explicit sparse and structured representation of $Z$ can be determined.

Consequently, the Hessian of the objective is diagonal and the reduced Hessian of the objective is tridiagonal. However, the matrix of interest is the reduced Hessian of the Lagrangian function. The Hessian of the weighted sum of the constraint function has a block structure. The $(1,1)$ and $(2,2)$ blocks are the zero matrix and the $(2,1)$ and $(1,2)$ are diagonal matrixes. Again it can be shown that the cross-product with $Z^{T}$ and $Z$ results in tridiagonal matrices, which implies the reduced Hessian of the Lagrangian is tridiagonal. This further implies we need not assume the reduced Hessian is small and the cost of its factorization also increases linearly. 


\section{Results}

\subsection{Verification of results from standard utilities}

Research in finance and economics has primarily focused on utilities in the normative class [34, $29,12,9]$. In order to perform a check of our modeling and solution method, we applied our method to utilities in the normative class and checked if our results qualitatively match those of others. We start with a constant relative risk aversion utility. The return parameters for the risky asset are chosen to be such that the mean and variance of the yearly return matches that of long-term (25 years) average yearly returns of a stock index, such as S\&P 500. The 'up' outcome risky asset return is $S_{u}=1.25$ and the 'down' outcome is $S_{d}=0.95$. The riskfree asset is a money market account yielding a yearly return of 1.05.

\subsubsection{Logarithmic Utility}

Absolute risk aversion, $A(W)$ as defined by Arrow and Pratt [13] is given as $\frac{-U^{\prime \prime}(W)}{U^{\prime}(W)}$, for twice continuously differentiable utilities. It is a measure of aversion to risk for different values of wealth. While, relative risk aversion, $R(W)$ is defined as $\frac{-W * U^{\prime \prime}(W)}{U^{\prime}(W)}$, and is a measure for aversion to risk relative to the wealth level (see Huang and Litzenberger [13]). The relative risk aversion is a constant for constant relative risk aversion(CRRA) utilities, i.e. risk aversion decreases with wealth.

Logarithmic utility is a constant relative risk aversion utility. In continuous time, given two asset classes with prices governed by a constant coefficient stochastic differential equation, the optimal allocation is given as : $X_{t}^{*}=\frac{\alpha-1}{\sigma^{2}(1-\gamma)}$, where $\alpha, \sigma$ are coefficients governing the stochastic differential equation for asset-price dynamics and $1-\gamma$ is the relative risk 

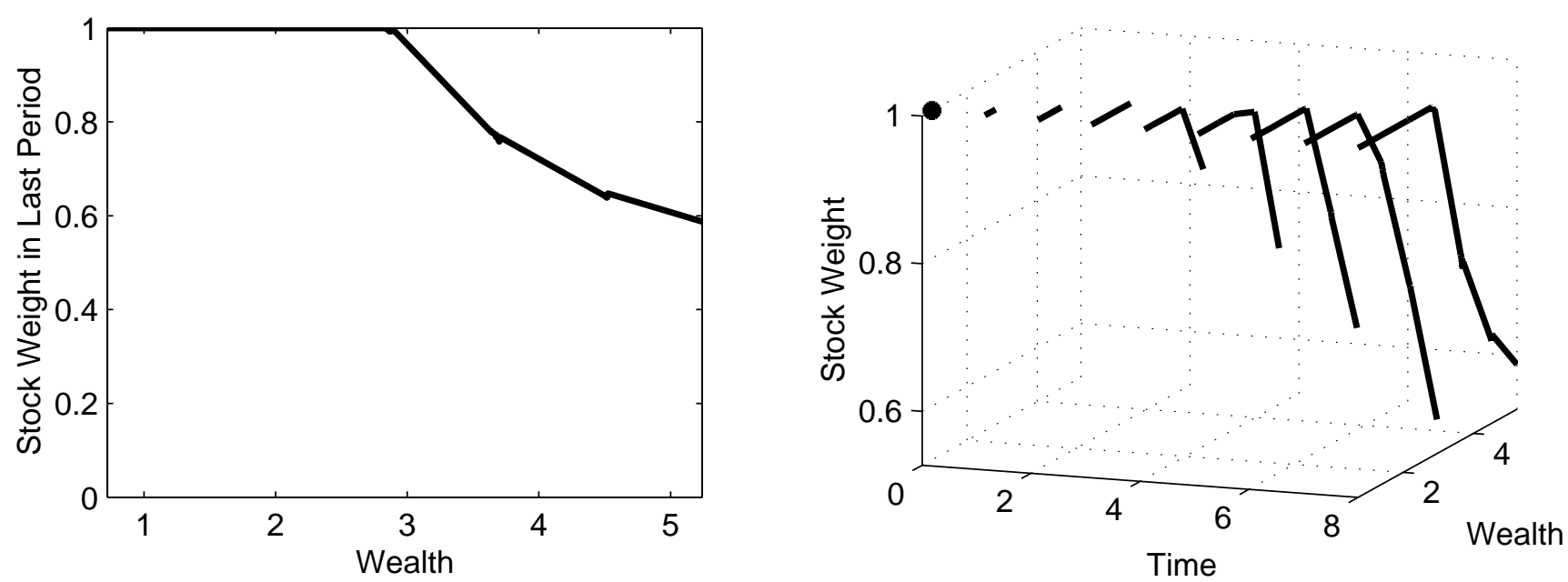

Figure 2: Optimal Stocks investment weights for a 9 periods problem using Negative Exponential utility, plotted by wealth levels at nodes for each time period.

aversion (see Merton [29]). Therefore, the optimal allocation for the logarithmic utility is a constant over time. Optimal allocation from our computations is a constant over all time-periods, $X_{t, n}=1$ for all $t$ and $n$.

\subsubsection{Negative Exponential Utility}

The exponential utility belongs to the constant absolute risk aversion class (CARA), which implies that with increasing level of wealth the aversion to risk remains the same. The utility asymptotes to a horizontal line as wealth goes to infinity. The continuous time result for the Negative Exponential utility is $X_{t}^{*}=\frac{\alpha-1}{\eta r \sigma^{2} W_{t}}$, where $\eta$ is the absolute risk aversion. Therefore, the optimal dollar amount invested in stocks is a constant. Our results for a 9 periods problem are in figure 2. The optimization is done with constraints that allow no short selling, hence the saturation at low wealth levels. 


\subsection{Optimal results for behavioural utilities}

Real decision-makers are not economic automatons, they are from time to time affected by emotions and cognitive hindrances in making rational decisions. For these reasons their utility functions may differ from the ones implied by the normative theory of choice. In what follows it is assumed that although this may be the case, the decision-makers should nevertheless make decisions that are optimal with respect to their utilities. Throughout this section wherever relevant, it is assumed that the decision-maker is an expected utility maximizer.

The optimization problem formulated in the previous section, with binomial-tree model for asset-returns, is to find the optimal investment decision variables, $X$, so that the expected utility of wealth at the planning horizon is maximized, i.e.,

$$
\begin{gathered}
\max _{X} \sum_{m=2^{T-1}}^{2^{T}-1}\left[p * U\left(X_{T-1, m} * W_{T-1, m} * S_{u}+\left(1-X_{T-1, m}\right) * W_{T-1, m} * R_{f}\right)+\right. \\
\left.p * U\left(X_{T-1, m} * W_{T-1, m} * S_{d}+\left(1-X_{T-1, m}\right) * W_{T-1, m} * R_{f}\right)\right] \\
\ni 0 \leq X \leq e,
\end{gathered}
$$

where $p$ is the probability of each node at the planning horizon, $e$ is vector of 1 's, $T$ is the planning horizon and $U($.$) is the investor's utility for wealth at the planning horizon. The$ index $m$ ranges over all the outcomes at one period prior to the planning horizon. In our problem formulation we permit no short sale, i.e. borrowing of assets is not allowed. This restriction translates to the above bounds on the decision variables. These bounds reflect regulatory constraints on positions the planner can take on the asset classes. It would not alter the nature of the problem if short selling was allowed. 
In the above formulation it is possible to solve for the optimal asset allocation strategy for a large class of utilities, only some of these are presented in what follows. Also, the size of problems for which results are presented is chosen to be moderate for ease of presentation. The results from non-uniform time-periods framework is presented only for the loss-aversion utility.

\subsubsection{Loss Aversion Utility}

Tversky and Kahnemann (see $[18,41]$ for details) developed an alternative theory to the expected utility theory of normative choice, called the Prospect theory. Essential characteristics of the Prospect theory utility are :

Reference dependence Gains and losses defined relative to a known reference point are the primary carriers of utility. People perceive and evaluate different choice options as a gain or a loss from a reference point.

Loss aversion Losses loom larger than corresponding gains resulting in the utility function to be steeper in the negative than in the positive domain. An amount of loss elicits more 'unhappiness' than the same amount of gain elicits 'happiness.'

Diminishing sensitivity Gain or loss of an extra dollar results in a lesser impact as the amount of overall gain or loss gets larger.

These characteristics imply that the behavioral utility function has an asymmetric S-shape, that is concave above a reference point and convex below it (see figure 3). A steep decrease in utility below the reference point captures the loss aversion behaviour. For brevity we will 


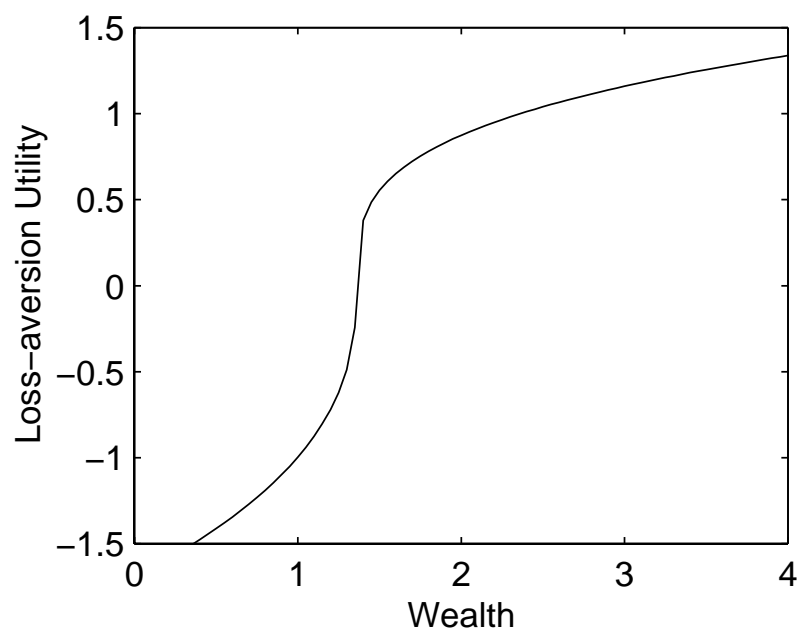

Figure 3: Plot of Prospect theory loss-aversion utility against wealth, W.

refer to this utility as loss-aversion utility. Lack of concavity and differentiability (at the reference point) of the loss-aversion utility distinguish it from the traditional utility functions and makes computation of the corresponding optimal investment decision more challenging.

Figures 4 and 5 give the optimal asset allocation for loss-aversion utility for $T=14$, where the reference point in the loss-aversion utility is taken as an $8 \%$ annual return on the starting wealth. Note that this problem has 32,766 variables. In figure 4(i) the optimal allocation for nodes at the period prior to planning horizon are plotted against wealth levels. Figure 5(ii) is a similar three dimensional plot for all the time periods of the 14 period problem. From figure 4 it is clear that investment weights are wealth dependent. As one nears the planning horizon, for low wealth levels the decision is to invest in the risky asset. For medium wealth level, investment is also done in riskfree asset as compared to high wealth levels, where investment is again largely in the risky asset. This is markedly different from the result for the CARA and CRRA normative utilities seen before. Note that wealth levels at several 

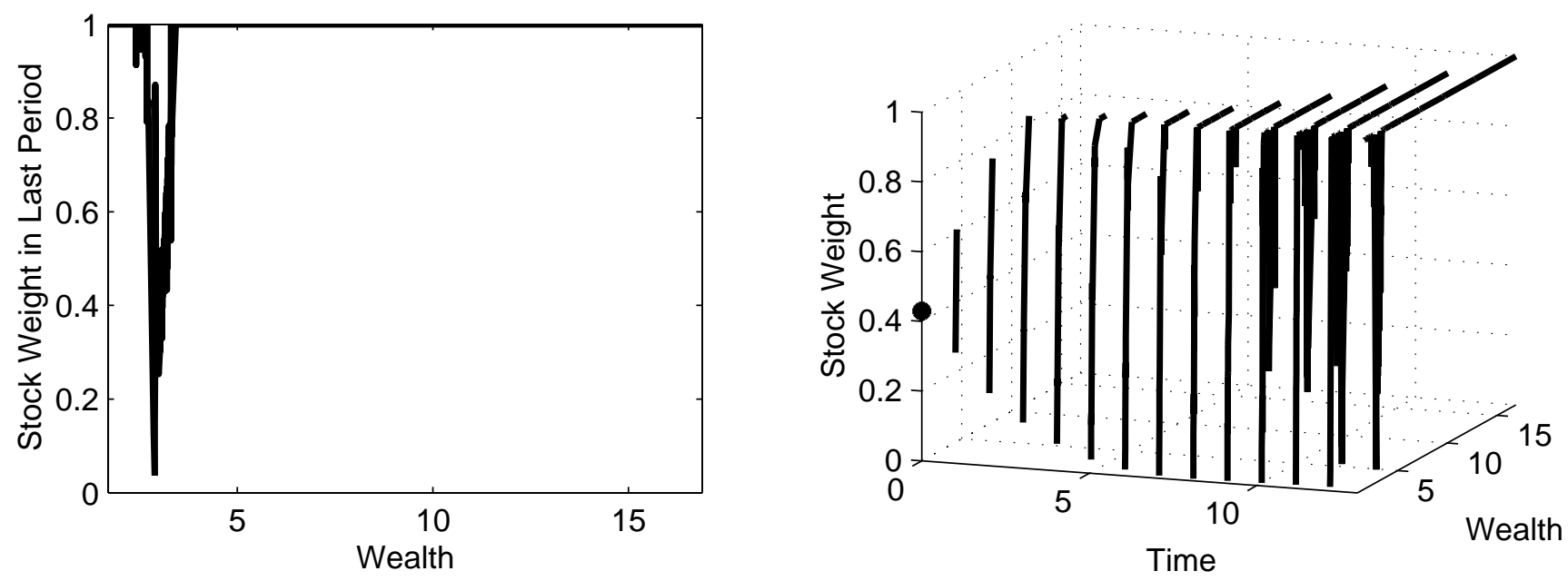

Figure 4: Optimal Stocks investment weights for a 14 periods problem using Loss aversion utility, plotted by wealth levels (i) for $t=13$ and (ii) for nodes for each time period. nodes at a time period coincide. In order to illustrate this point, in figure 5 the frequency of wealth levels is plotted along with investment weights for $t=13$.

Choice of the reference point is likely to affect the optimal asset allocation. We vary the reference point and obtain optimal investment decisions for loss-aversion utility. This provides insight into sensitivity to ones reference for evaluation of portfolio performance. In figure 6 the optimal asset allocation for a different reference point for the loss-aversion utility is given. The first reference point is as before, the second corresponds to the riskfree annual rate of return of $5 \%$. The $\mathrm{V}$-shaped dip in allocation becomes deeper and shifts with the lowering of the reference point.

Note that in the above formulation of the problem all the characteristics of prospect theory modeling of utility are not followed, in particular, we do not weight the probabilities associated with the final nodes in the tree according to Kahnemann-Tversky's prospect 


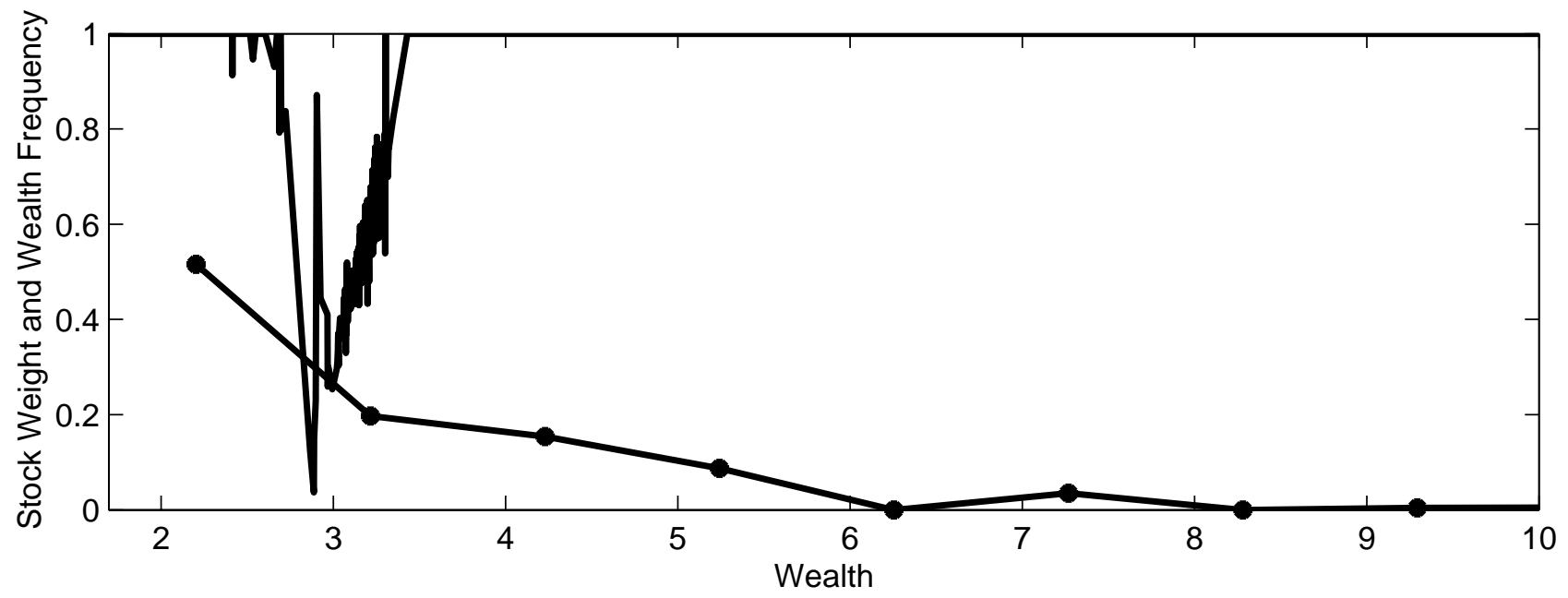

Figure 5: Optimal Stocks investment weights for a 14 periods problem using Loss aversion utility, against the frequency plot for wealth levels at $t=13$.

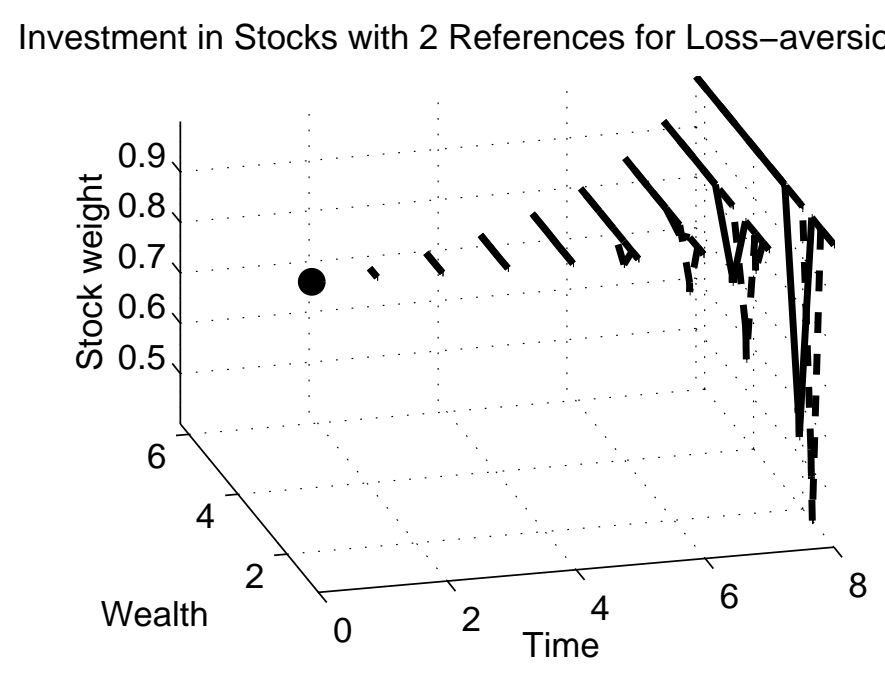

Figure 6: Optimal Stocks investment weights for a 9 periods problem using Loss aversion utility, with different reference points (continuous for $8 \%$ yearly return, dashed line for $5 \%$ yearly return). 


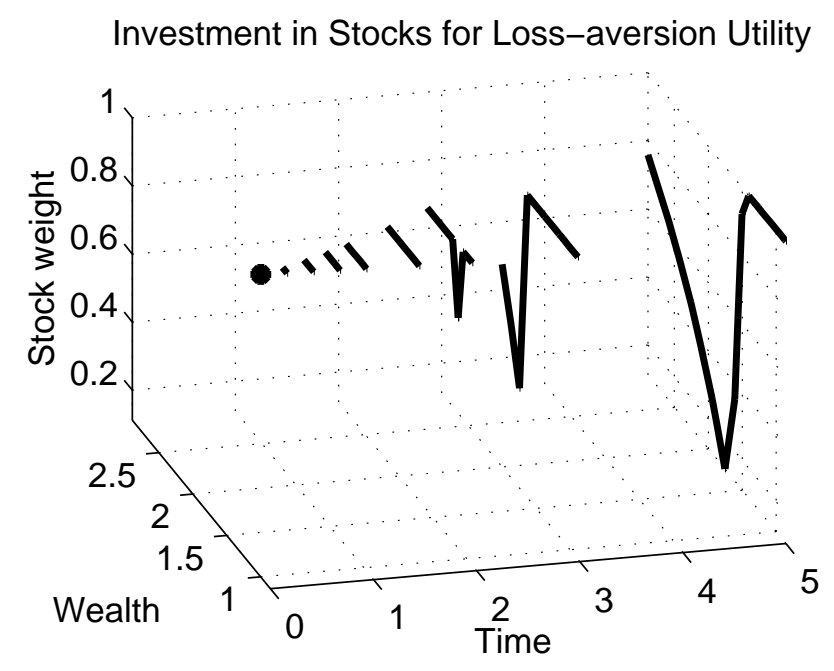

Figure 7: Optimal Stocks investment weights for a 9 periods problem using Loss aversion utility, with non-uniform time periods (4 quarters, 2 half year, 1 one year, 1 two year and 1 four year periods).

theory. In the current formulation this cannot be done, since probability of each outcome at any time is the same. Modifications to allow this nonlinear transformation of probabilities can be done without increasing the problem complexity by much.

As described in section 3.2.2, in this framework we allow for non-uniform time periods. Results for a non-uniform time periods problem are given in figure 7. A 9 periods problem is solved with 4 quarters, 2 half-year periods, 1 one year period, 1 two year period and 1 four year period. Therefore, a total of 9 years is spanned with a higher resolution for periods close to $t=0$. The nature of the results is similar to the uniform periods case, at times closer to planning horizon there is a 'dip' in risky asset allocation for a range of wealth level. 


\subsubsection{Piece-wise Linear Utility}

The piece-wise linear utility is also a utility with a reference point, but unlike the lossaversion utility, there is a kink at the reference point. The slope of the linear is higher below the reference point than above it. As the piece-wise linear utility is not strictly convex below the reference point, it is not strictly risk seeking for low levels of wealth.

It is more efficient to first alter the formulation of the problem to remove the discontinuity. The new formulation is as follows. The wealth levels at all nodes and times are taken as variables, including the wealth at the planning horizon $T$. Two extra variables are defined for each node at the planning horizon of the tree, i.e. there are now $\left(2^{T}-1\right) X$ variables, $\left(2^{T+1}-1\right) W$ variables and $2 * 2^{T}$ additional variables. Let these additional variables be $Z_{1}\left(1: 2^{T}\right)$ and $Z_{2}\left(1: 2^{T}\right)$. If the slope of each linear in the utility is $c_{1}$ and $c_{2}$ and the reference point is $\hat{w}$, then the objective for the new, equivalent problem formulation becomes

$$
\begin{gathered}
\max _{X, W, Z_{1}, Z_{2}} \sum_{i=1}^{2^{T}} \quad p *\left(c_{1} * Z_{1}(i)+c_{2} * Z_{2}(i)\right) \\
\ni W_{T, 2^{T}+i-1}=Z_{1}(i)+Z_{2}(i) \text { for } i=1: 2^{T} \\
0 \leq Z_{1}(i) \leq \hat{w} \text { for } i=1: 2^{T} \\
0 \leq Z_{2}(i) \leq \infty \text { for } i=1: 2^{T},
\end{gathered}
$$

along with the constraints (1) \& (2) involving the $X$ and $W$ variables. The constraints (4), (5) \& (6) ensure that the variables $Z_{1}\left(1: 2^{T}\right)$ and $Z_{2}\left(1: 2^{T}\right)$ are consistent with the variables $W_{T, n}$ for $n=2^{T}: 2^{T+1}-1$. In the new formulation the objective is linear.

It may be thought that increasing the number of variables in this manner would have a serious impact on the efficiency of the solver. However, at every iteration it can be shown 

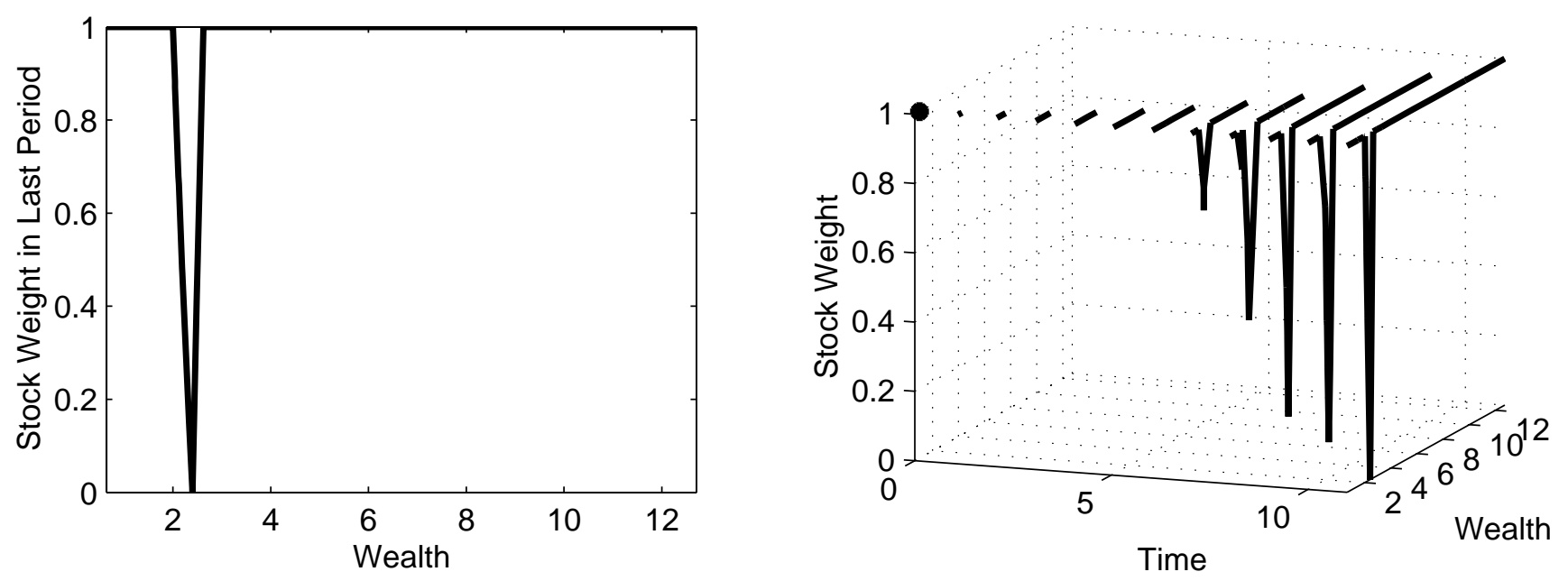

Figure 8: Optimal Stocks investment weights for a 12 periods problem using Piece-wise Linear utility, plotted by wealth levels (i) for $t=11$ and (ii) for nodes for each time period. that at least one of the two variables must be on a bound. Consequently, the degrees of freedom at each iteration does not increase and it is this factor that dominates the efficiency of SNOPT. In figure 8 the optimal asset allocation is given for $T=12$. The pattern for investments is quite similar to the Loss aversion utility. We also compared the solutions for shifted reference points, taking them to be the starting wealth and riskfree return on the starting wealth. Since these results are similar to the Loss aversion utility case, we don't display them for shifted reference point.

\subsubsection{Probability of reaching a Goal}

This utility evaluates the performance of the wealth accumulated at the planning horizon by the probability of it exceeding a prespecified target wealth level, referred to as 'Goal.' The decision-makers may come up with a target wealth level by estimating the total expenses they expect to have after retirement. Because of its intuitive appeal, financial advisors like to 
talk in terms of this utility with their client. The utility may be written as $\operatorname{Prob}\left(W_{T}>G\right)$, where $W_{T}$ is the random variable denoting the wealth at the planning horizon and $G$ is the target wealth level.

In a discrete model for asset-returns this utility is a discontinuous one, hence the problem needs to be formulated so that the objective becomes a continuous function. Our algorithmic approach is as follows.

\section{Algorithm:}

Adopt an investment strategy such that the target value, 'Goal,' is achieved at a certain number of nodes in the planning horizon.

While An additional node can achieve the Goal

Select a node at the planning horizon with the largest wealth less than the Goal level. Optimize the wealth level at the selected node, along with constraints that keep the Goal nodes to stay at the Goal level.

\section{End}

Ties in node selection are broken by selecting a lower numbered node in the ordering of nodes in figure 1. This formulation of the problem results in the objective being continuous in each optimization problem solved in the algorithm.

A good starting point is crucial in this problem. We present two strategies to get a good starting point. 
Strategy 1 The investor initially invests only in the risky asset unless a transition node is reached. At the transition node part of wealth is invested in the riskfree asset and at all subsequent nodes all wealth is invested in the riskfree asset. The determination of the transition node and the proportion of wealth to invest in the risky asset is determined by assuring the target wealth is reached at the node at the end of the planning horizon.

Strategy 2 The investor initially invests both in risky and riskfree asset unless a transition node is reached. At the transition node investment in the two assets is made such that at all subsequent nodes wealth needs to be invested only in the riskfree asset to achieve the target wealth. Prior to the transition node investment in the two asset classes is made so that at each node the expected returns for the remaining time periods just attains the 'Goal' level.

Note that both these starting points are feasible and that the total number of active constraints is close to the number of variables. After adopting one of the above two strategies a set of nodes at the planning horizon are at the 'Goal' level. We next pick the highest nonGoal node and maximize the wealth level at this node along with constraints that the nodes that have already achieved the 'Goal' stay at 'Goal.' Optimization could be done on a subtree or on the entire tree. In figure 9 investments for a planning horizon, $T=7$ are given. For low levels of wealth, the investment is in the risky asset, but with increasing wealth as meeting the 'Goal' gets more likely, investment increases in riskfree asset.

It is interesting to observe the number of nodes at the planning horizon at which 'Goal' is attained for different values of 'Goal' levels. Figure 10 gives a plot of number of nodes at which 'Goal' is attained against different 'Goal' levels. The discrete nature of the problem 

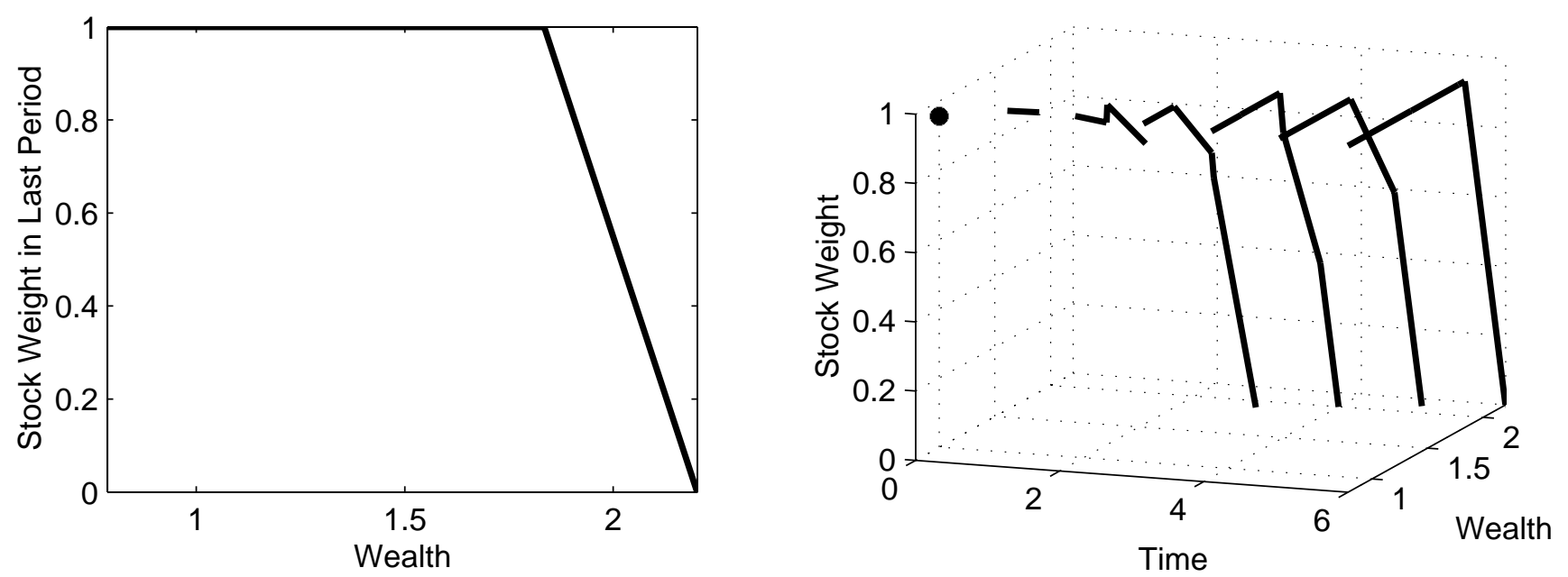

Figure 9: Optimal Stocks investment weights for a 7 periods problem using Probability of Goal utility, plotted by wealth levels (i) for $t=6$ and (ii) for nodes for each time period. results in the curve in figure 10 not being smooth. Note the number of conspicuous jumps in the number of Goal-nodes for the 'Goal' values such as, for 2.23, 2.51, 3.4. While this is partially an artifact of a discrete-outcome tree, it indicates the importance of sensitivity analysis with respect to the set 'Goal.'

A variant of the above problem is that the investor has a rough idea of what their 'Goal' value is, but would like to maximize the 'Goal' value once a certain number of nodes at which it is attained has been determined. For this problem we again adopt one of the above two strategies and perform the above mentioned optimization on the number of nodes that attain the 'Goal.' We then maximize the 'Goal' value itself, under constraints that the number of nodes that attained the 'Goal' does not decrease. 


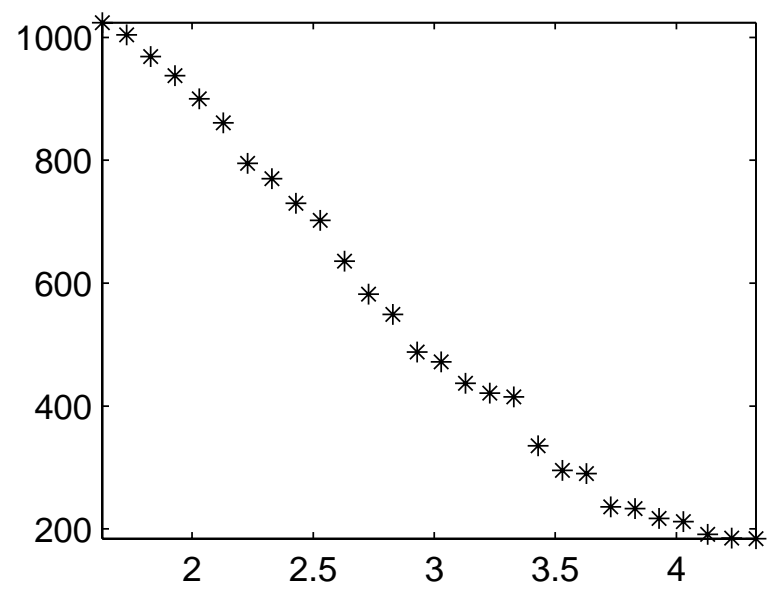

Figure 10: Number of nodes at which target wealth is achieved for a range of target wealth values.

\subsubsection{Variant of Probability of reaching a Goal}

Variant of the previous utility arise when the investor also cares for the downside risk besides caring for reaching a target wealth level. As before, in a discrete model for asset-returns this utility is a discontinuous one, hence the problem needs to be formulated so that the objective becomes a continuous function. We adopt a similar strategy to that described earlier; adopt an investment strategy such that the target value, 'Goal,' is achieved at certain number of nodes at the planning horizon, along with no node being below a lower bound, say $L$.

A good starting point is again crucial. We modify the two strategies from the previous section for this problem as

Strategy 3 Following strategy 1 the investor initially invests only in the risky asset unless a transition node is reached. At the transition node part of wealth is invested in the riskfree asset and at all subsequent nodes all wealth is invested in the riskfree asset. Further on the downside, the node where the wealth level becomes low enough that 
investing in the riskfree asset will barely make it stay above $L$ at the planning horizon, the investor invests only in the riskfree asset in all subsequent nodes.

Strategy 4 Following strategy 2 the investor initially invests both in risky and riskfree asset unless a transition node is reached. The strategy is then identical to that of strategy 3.

Therefore, there are now two types of transition nodes, one is identical to that described in strategy 1 and the other is when continuing investing in the risky asset no longer ensures the final wealth satisfies its lower bound. As with the first type of transition node, the investment is split and at all subsequent nodes all investment is in the riskfree asset.

After adopting one of the above two strategies a set of nodes at the planning horizon are at the 'Goal' level and none of them is below $L$. We next pick the highest non-Goal node and maximize the wealth level at the node along with constraints that the nodes that already achieved the 'Goal' stay at 'Goal' and none goes below L. Optimization could be done on a sub-tree or on the entire tree. The outcome of this procedure is an investment strategy similar to the previous Probability of reaching a 'Goal' utility, but with investments shifting to riskfree asset also for low levels of wealth. Therefore, this strategy is quite the opposite of that for Loss-aversion utility.

As before, another variant of the above problem is that the investor would like to maximize the 'Goal' value once a certain number of nodes at which it is attained is figured out. For this problem we again adopt one of the above two strategies and perform the above mentioned optimization on the number of nodes that attain the 'Goal.' We then maximize the 'Goal' value itself, under constraints that the nodes that attained the 'Goal' stay so and 
no node at the planning horizon goes below the level $L$.

\section{Conclusion}

In this article we have developed a framework for obtaining long-term investment decisions for a more general class of utilities than those satisfying the axioms of choice. A distinction between what are called the behavioural utilities and the normative utilities is made on the basis of whether the decision-makers preferences satisfy all the axioms of choice. If it happens that some of the axioms of choice are not satisfied, the resulting utility is less wellbehaved, making the computation of the optimal investment decisions more challenging. In the framework developed in this article it is possible to solve for the optimal investment decisions for these utilities.

Our objective was to develop a model with considerable flexibility. One consequence is that this leads to a large nonlinearly constrained optimization problem. The optimization problem has a structured and a sparse constraint Jacobian. We use this characteristic to our computational advantage by using the SNOPT software package. Further, the number of constraints and bounds are comparable to the number of variables, as a result even though there may be tens of thousands variables in the problem, the number of free variables is manageable. This is another feature that helps improve SNOPT's efficiency. One advantage of using a general-purpose algorithm is that it allows a great degree of model flexibility and additionally, such algorithms will continue to develop and hence, offer continued improved performance.

We solved the problem for several utilities, starting with some from the normative class. 
Our results for these utilities are consistent with those found in the literature. The results for the behavioural utilities indicate an interesting dependence of the strategy on the wealth levels. For reference point based utilities, the investment strategy for each time period recommends to be fully invested in the risky asset for low and high levels of wealth, whereas in a certain medium range of wealth recommends to deviate from fully investing in the risky asset. This medium range of wealth, where investment should also be made in the riskfree asset, shifts with the reference point of the utility. The investment decisions for the probability of reaching a pre-specified 'Goal' utility resemble that of the negative exponential utility, decreasing investment in the risky asset at higher levels of wealth. However, for the utility where along with reaching a 'Goal' the downside risk also matters, positions in the risky asset get smaller also for the lower levels of wealth.

We provide for solving the problem with non-uniform time periods. This formulation allows for a finer time-refinement for periods closer to the beginning of the planning period. This feature is particularly attractive if the problem is solved on a rolling basis. In most retirement funds periodic contributions are made to the fund, our modeling approach allows for incorporating this in the model.

\section{Extensions}

The framework developed in this article for solving the long-term financial planning problem is very flexible. We can incorporate multiple asset classes, multiple branching at nodes, correlation in the returns of the asset classes, different patterns in the contribution schemes, to name only a few. Additional asset classes or correlation in their returns will increase 
the size of the problem, however the structure and sparsity of the problem will be retained. Instead of maximizing the utility of wealth at the planning horizon, utility of wealth at the interim time periods can also be included in the objective, potentially with varying reference points in the utility. Additionally, considering transaction costs and tax related issues is also an important extension of the problem.

In the solution method we are currently unable to use the exact Hessian information for computing the search direction, even though computing it exactly is possible for our formulation of the problem. However, algorithms that incorporate second derivative are under development and will be available shortly. Being able to use this exact information will give us greater efficiency and ability to solve much larger problems.

The solution for several of the utilities considered suggests a structure in the solution. After further confirmation of these structures, they may be used to 'decompose' the problem, which will in turn aid in solving larger problems.

\section{Acknowledgements}

It is a pleasure for us to thank Professor William F. Sharpe for introducing this problem to us and for his encouragement in seeking a solution. This paper was materially improved by a very perceptive referee. We are thankful for the care and effort taken by this referee. We are indebted to both the referees for numerous suggestions for improvement in the presentation. 


\section{References}

[1] David E. Bell, Howard Raiffa, and Amos Tversky. Decision Making - Descriptive, Normative, and Prescriptive Interactions. Cambridge University Press, Cambridge, U.K., 1988.

[2] Shlomo Benartzi and Richard H. Thaler. Myopic loss aversion and the equity premium puzzle. Quarterly Journal of Economics, 110(1):75-92, 1992.

[3] John R. Birge. Current trends in stochastic programming computation and applications. www.iems.northwestern.edu/ jrbirge/Public/html/algor/stojoc.ps, 1995.

[4] John R. Birge. Stochastic programming computation and applications. INFORMS Journal on Computing, 9(2):111-133, 1997.

[5] John R. Birge and Francois Louveaux. Introduction to Stochastic Programming. Springer Verlag, 1997.

[6] C. Bischof, P. Khademi, A. Mauer, and A. Carle. ADIFOR 2.0: automatic differentiation of fortran 77 programs. IEEE Computational Science and Engineering, 3(3):18-32, 1996.

[7] Zvi Bodie, Robert C. Merton, and William F. Samuelson. Labor supply flexibility and portfolio choice in life-cycle mode. Journal of Economic Dynamics and Control, 16:427-449, 1992.

[8] Michael J. Brennan, Eduardo S. Schwartz, and Ronald Lagnado. Strategic asset allocation. Journal of Economic Dynamics and Control, 21:1377-1403, 1997. 
[9] John Y. Campbell and Luis M. Viciera. Consumption and portfolio decisions when expected returns are time varying. NBER Working paper No. 585\%, 1996.

[10] John Cox, Stephen Ross, and Mark Rubinstein. Option pricing: A simplified approach. Journal of Financial Economics, 7(3):229, 1979.

[11] George B. Dantzig and Gerd Infanger. Multi-stage stochastic linear programs for portfolio optimization. Annals of Operations Research, 45:59-76, 1993.

[12] Darrell Duffie and Thaleia Zariphopoulou. Optimal investment with undiversifiable income risk. Mathematical Finance, 3(2):135-148, 1993.

[13] Chi fu Huang and Robert H. Litzenberger. Foundations for Financial Economics. Prentice Hall, Inc., Englewood Cliffs, New Jersy, 1988.

[14] Philip E. Gill, Walter Murray, and Michael A. Saunders. User's Guide for SNOPT(Version 4.0): A Fortran Package for Sparse Nonlinear Programming. Technical Report SOL 86-2, 1998.

[15] Philip E. Gill, Walter Murray, and Michael A. Saunders. SNOPT: An SQP algorithm for large-scale constrained optimization. SIAM Journal on Optimization, 12(4):979-1006, 2002.

[16] Philip E. Gill, Walter Murray, and Margaret H. Wright. Practical Optimization. Academic Press, Harcourt Brace and Company, London, 1981.

[17] Gerd Infanger. Planning Under Uncertainty - Sovling Large-Scale Stochastic Linear Programs. Boyd \& Fraser Publishing Company, Danvers, Massachusetts, 1994. 
[18] Daniel Kahneman and Amos Tversky. Prospect theory: An analysis of decisions under risk. Econometrica, 47:313-327, 1979.

[19] Ioannis Karatzas, John P. Lehoczky, Suresh Sethi, and Steven E. Shreve. Explicit solution of a general consumption/investment problem. Mathematics of Operations Research, 11(2):261-294, 1986.

[20] Roy Kouwenberg, Arjan Berkelaar, and Thierry Post. Optimal portfolio choice under loss aversion. Working Paper, 2003.

[21] Roy Kouwenberg and Stavros Zenios. Stochastic programming models for asset liability management. Handbook of Asset and Liability Modeling in series North-Holland Handbooks in Finance, 2001.

[22] David I. Laibson. Comments on personal retirement saving programs and asset accumulation: Reconciling the evidence. Studies in the Economics of Aging, NBER Project Report, W5599:106-124, 1998.

[23] David I. Laibson, Andrea Repetto, and Jeremy Tobacman. Self-control and saving for retirement. Brookings Papers on Economic Activity, 1:91-196, 1998.

[24] D. Lamberton and B. Lapeyre. Introduction to Stochastic Calculus Applied to Finance. Chapman and Hall, London, UK, 1996.

[25] David G. Luenberger. Investment Science. Oxford University Press, New York, 1998.

[26] Andreu Mas-Colell, Michael D. Winston, and Jerry R. Green. Microeconomic Theory. Oxford University Press, New York, 1995. 
[27] Robert C. Merton. Lifetime portfolio selection under uncertainty: The continuous-time case. Review of Economics and Statistics, 51(3):247-257, 1969.

[28] Robert C. Merton. Optimum consumption and portfolio rules in a continuous-time model. Journal of Economic Theory, 3(4):373-413, 1971.

[29] Robert C. Merton. Continuous-Time Finance. Basil Blackwell Inc., Cambridge, Massachusetts $02142,1990$.

[30] John M. Mulvey, Daniel P. Rosenbaum, and Bala Shetty. Strategic financial risk management and operations research. European Journal of Operations Research, 97:1-16, 1997.

[31] James M. Poterba, Steven F. Venti, and David A. Wise. Personal retirement saving programs and asset accumulation: Reconciling the evidence. NBER Working Paper, W5599, 1997.

[32] Sheldon M. Ross. Stochastic Processes. John Wiley \& Sons, Inc., New York, 1996.

[33] Ariel Rubinstein. Modeling Bounded Rationality. MIT Press, Cambridge, MA, 1997.

[34] Paul A. Samuelson. Lifetime portfolio selection by dynamic stochastic programming. Review of Economics and Statistics, 51:239-246, 1969.

[35] William F. Sharpe, Gordon J. Alexander, and Jeffery V. Bailey. Investments. Prentice Hall, Inc. Englewood Cliffs, New Jersey, 1995.

[36] John B. Shoven. The location and allocation of assets in pension and conventional savings accounts. NBER Working Paper No. 7007, 1999. 
[37] Herbert A. Simon. Models of Bounded Rationality vol. I, II. MIT Press, Cambridge, MA, 1982.

[38] Richard H. Thaler, Amos Tversky, Daniel Kahneman, and Alan Schwartz. The effect of myopia and loss aversion on risk taking: an experimental test. Quarterly Journal of Economics, 5:297-323, 1992.

[39] Amos Tversky. Additivity, utility an subjective probability. Journal of Mathematical Psychology, 4:175-202, 1967.

[40] Amos Tversky and Daniel Kahneman. Loss aversion in riskless choice: A referencedependent model. Quarterly Journal of Economics, 106(4):1039-1061, 1991.

[41] Amos Tversky and Daniel Kahneman. Advances in prospect theory: Cumulative representation of uncertainty. Journal of Risk and Uncertainty, 5:297-323, 1992.

[42] Hal R. Varian. Microeconomic Theory. W.W. Norton and Company, Inc., New York, 1992.

[43] Luis M. Viciera. Optimal Consumption and Portfolio Choice for Long-Horizon Investors. PhD thesis, Department of Economics, Harvard University, 1998.

[44] George Wright. Behavioral Decision Making. Plenum Press, New York, N.Y., 1985. 